\title{
Treatment with Diazepam in Acute Stroke Prevents Poststroke Seizures: A Substudy of the EGASIS Trial
}

\author{
Julia H. van Tuijla, b Elisabeth P.M. van Raak ${ }^{a} \quad$ Robert J. van Oostenbrugge $^{a, c}$ \\ Albert P. Aldenkamp a, c, d Rob P.W. Rouhla, c, d \\ aDepartment of Neurology, Maastricht University Medical Center, Maastricht, The Netherlands; ${ }^{b}$ Department of \\ Neurology, Elisabeth TweeSteden Hospital, Tilburg, The Netherlands; 'School for Mental Health \& Neuroscience, \\ Maastricht University, Maastricht, The Netherlands; ${ }^{\mathrm{d} A c a d e m i c}$ Center for Epileptology Kempenhaeghe and \\ Maastricht University Medical Center, Maastricht, The Netherlands
}

\section{Keywords}

Stroke · Seizures · Epilepsy · Diazepam · Antiepileptic drugs

\section{Abstract}

Objective: The frequency of seizures after stroke is high, with a severe impact on the quality of life. However, little is known about their prevention. Therefore, we investigated whether early administration of diazepam prevents the development of seizures in acute stroke patients. Methods: We performed a substudy of the EGASIS trial, a multicenter double-blind, randomized trial in which acute stroke patients were treated with diazepam or placebo for 3 days. Follow-up was after 2 weeks and 3 months. The occurrence of seizures was registered prospectively as one of the prespecified secondary outcomes. Results: $784 \mathrm{EGASIS}$ patients were eligible for this substudy (389 treated with diazepam [49.6\%] and 395 treated with placebo [50.4\%]). Seizures were reported in 19 patients (2.4\% of the total patient group). Seizures occurred less frequently in patients treated with diazepam (1.5 vs. $3.3 \%$ in the placebo group); however, this difference was only statistically significant in patients with a cortical ante- rior circulation infarction $0.9 \%$ in the diazepam group vs. $4.6 \%$ in the placebo group, incidence rate ratio $0.20,95 \% \mathrm{Cl}$ : $0.05-0.78, p=0.02$, NNT $=27$ ). Conclusion: We found that a 3-day treatment with diazepam after acute cortical anterior circulation stroke prevents the occurrence of seizures in the first 3 months following stroke.

(C) 2021 The Author(s)

Published by S. Karger AG, Basel

\section{Introduction}

The frequency of seizures after stroke is rather high and varies between 0.3 and $13.6 \%$ [1]. Poststroke seizure risk depends on stroke severity, lesion size, stroke type, cortical involvement, and stroke recurrence. Seizures have a negative impact on stroke patients. They relate to a worse neurological outcome and higher mortality in the short term [2-6]. Long-term consequences are, amongst others, loss of independence, impaired self-confidence, and a higher risk of fractures after falling [7-9]. Prevention of seizures would therefore be worthwhile. In animal studies, several antiepileptic drugs have shown antiepi-

karger@karger.com www.karger.com/ced

Karger $\stackrel{\text { ' }}{5}$

BOPEN ACCESS
(C) 2021 The Author(s)

Published by S. Karger AG, Basel

This is an Open Access article licensed under the Creative Commons Attribution-NonCommercial-4.0 International License (CC BY-NC) (http://www.karger.com/Services/OpenAccessLicense), applicable to the online version of the article only. Usage and distribution for commercial purposes requires written permission.
Rob P.W. Rouhl

Department of Neurology

Maastricht University Medical Centre

PO Box 5800, NL-6202AZ Maastricht (The Netherlands)

R.Rouhl@mumc.nl 
leptogenic properties $[10,11]$. In humans, evidence is sparse, with some observational studies and only 1 randomized clinical trial is available [12-16]. Unfortunately, we had to terminate a randomized clinical trial, in which the early prophylactic use of levetiracetam following stroke was investigated, due to a low inclusion rate [17]. Thus, the prophylactic use of antiepileptic drugs after stroke is still controversial $[15,18]$. Therefore, we aimed to generate new insight by presenting the effect of a 3-day treatment with diazepam on the occurrence of seizures in stroke patients. This is a substudy of the previously published EGASIS trial [19], in which diazepam was tested for its neuroprotective properties in stroke patients. The primary endpoints, functional outcome, as well as drug safety, did not differ between the diazepam and the placebo group. In the EGASIS trial, the occurrence of seizures was one of the prespecified secondary outcome measures. Since antiepileptogenic properties of diazepam have been shown in animal studies [11,20,21], we used the data from the EGASIS trial to test our hypothesis that diazepam has antiepileptogenic properties in patients.

\section{Methods}

The present study is a substudy of the EGASIS trial, a multicenter, randomized, placebo-controlled, double-blind, clinical trial studying the neuroprotective property and safety of diazepam after acute ischemic or hemorrhagic stroke [19].

\section{Patients}

Data from the EGASIS trial were used for this substudy. In the EGASIS trial, adult stroke patients were included within $12 \mathrm{~h}$ after stroke onset, with their diagnosis confirmed within 7 days by CT or MR imaging. Exclusion criteria were a clear indication for or a contraindication to benzodiazepines (at the discretion of the attending physician), unresponsive coma, total functional dependence before stroke, and participation in another neuroprotection trial. Current benzodiazepine use was not an exclusion criterion, but benzodiazepines had to be stopped before randomization. Randomization was carried out centrally by a 24 -h telephone service. All participants (patients, investigators, attending physicians, and nurses) were blinded to the allocated treatment.

After informed consent and randomization, patients were treated with 10-mg diazepam (or placebo) rectally administered as soon as possible after stroke onset, preferably within 3 but at most within $12 \mathrm{~h}$, followed by 10 -mg diazepam (or placebo) orally twice daily at 12-h intervals for the following 3 days (in total 6 doses of $10 \mathrm{mg}$ ) or until earlier discharge. Follow-up was after 2 weeks (or at earlier discharge) and 3 months.

For the EGASIS trial, the following baseline characteristics were assessed: age, sex, time between stroke onset and first trial medication, neurological status including alertness, blood pressure, and use of benzodiazepines before inclusion. For patients with ischemic stroke, stroke syndrome diagnosis was made according to the Oxfordshire Community Stroke Project classification [22]. This clas- sification uses clinical symptoms and signs to classify strokes as total anterior circulation infarction (TACI), partial anterior circulation infarction (PACI), lacunar infarction (LACI), and posterior circulation infarction (POCI). TACI and PACI are both cortical stroke syndromes of the anterior circulation.

At 2 weeks, follow-up information was gathered prospectively on the adequate use of trial medication, comedication, presence of epilepsy before stroke, cardiac status, vascular risk factors (known hypertension, myocardial infarction, angina pectoris, diabetes mellitus, prior stroke, and current smoking status), result of MRI or CT scan, length of hospital stay, and complications during hospital stay, explicitly including pneumonia and the occurrence of seizures after inclusion in the trial. All seizures recorded were diagnosed by the attending physician. Also, the modified Rankin score and Barthel index were recorded.

At 3 months of follow-up, the modified Rankin score, Barthel index, the occurrence of seizures, vital status (alive of dead), and place of residence, or when applicable, cause of death, were recorded. The diagnosis of seizures was left to the discretion of the attending physician who was blinded for the treatment allocation.

For the present substudy, we excluded patients from the EGASIS cohort with pre-existing epilepsy (to exclusively study the effects of the current cerebrovascular lesion on the development of seizures) and patients with a transient ischemic attack (as we wanted to examine the effect of diazepam on seizure development due to cerebral tissue damage). We used the prospectively recorded occurrence of poststroke seizures now as primary outcome measure and mortality as secondary outcome measure.

\section{Statistical Analysis}

As this study is an exploratory substudy on a prespecified secondary outcome measure of a randomized trial, there was no power calculation. We used intention-to-treat analyses in all patients and separately analyzed ischemic stroke and intracerebral hemorrhage patients. We compared baseline data with the Mann-Whitney $U$ test (for age) and Pearson $\chi^{2}$ test or Fisher's exact test (for the other variables). We compared the occurrence of seizures between the diazepam and the placebo group, while accounting for differences in exposure time with incidence rate ratios (IRR). We further examined the effects of diazepam in ischemic stroke patients in predefined subgroups of anterior and posterior circulation stroke (Oxfordshire Community Stroke Project classification). We predefined these groups because patients with cortical strokes have a higher seizure risk, and therefore, antiepileptogenic effects might be larger in patients with a cortical stroke. We expressed mortality risk association measurements with odds ratios (OR). We also examined mortality risks separately in relation to pneumonia. We considered 2 -tailed $p$ values of $<0.05$ statistically significant. We used IBM SPSS statistics version 21 and Stata/SE version 11.2 for statistical analyses.

\section{Results}

Initially, 880 patients were included in the EGASIS trial. One patient was excluded because of an unintentional double inclusion. Six patients did not have a stroke, 8 patients were lost to follow-up at 2 weeks, and another 22 
Table 1. Baseline characteristics for the patients at randomization

\begin{tabular}{lccc}
\hline Characteristics & $\begin{array}{c}\text { Diazepam } \\
n=389(\%)\end{array}$ & $\begin{array}{l}\text { Placebo } \\
n=395(\%)\end{array}$ & $p$ value \\
\hline Age, mean, SD & $70.53(12.1)$ & $70.73(11.9)$ & 0.899 \\
Age, median (range) & $72.5(21.0-96.9)$ & $72.8(20.7-95.5)$ & 0.899 \\
Male gender & $205(52.7)$ & $223(56.5)$ & 0.291 \\
Patient is alert & $338(86.9)$ & $339(85.8)$ & 0.664 \\
Treatment start <3 h & $78(20.1)$ & $72(18.2)$ & 0.516 \\
Treatment start 3-6 h & $159(40.9)$ & $161(40.8)$ & 0.974 \\
Treatment start 6-12 h & $152(39.1)$ & $162(41.0)$ & 0.580 \\
ICH & $43(11.1)$ & $48(12.2)$ & 0.631 \\
Ischemic stroke & $346(88.9)$ & $347(87.8)$ & 0.631 \\
$\quad$ POCI & $31(8.0)$ & $45(11.4)$ & 0.105 \\
$\quad$ LACI & $104(26.7)$ & $105(26.6)$ & 0.961 \\
$\quad$ PACI & $107(27.5)$ & $93(23.5)$ & 0.203 \\
$\quad$ TACI & $104(26.7)$ & $104(26.3)$ & 0.898 \\
Prior stroke & $75(19.3)$ & $74(18.7)$ & 0.846 \\
CE mechanism & $93(23.9)$ & $105(26.6)$ & 0.389 \\
Hypertension & $182(46.8)$ & $187(47.3)$ & 0.876 \\
Diabetes mellitus & $54(13.9)$ & $67(17.0)$ & 0.233 \\
Ischemic heart disease & $98(25.2)$ & $113(28.6)$ & 0.281 \\
Benzodiazepine use before inclusion & $46(11.8)$ & $44(11.1)$ & 0.763 \\
\hline
\end{tabular}

$\mathrm{SD}$, standard deviation; $<3 \mathrm{~h}$, within $3 \mathrm{~h}$ after stroke; ICH, intracerebral hemorrhage; POCI, posterior circulation infarction; LACI, lacunar infarction; PACI, partial anterior circulation infarction; TACI, total anterior circulation infarction; CE, cardioembolic.

patients were lost to follow-up at 3 months, leaving 843 patients in the trial of whom 426 were treated with diazepam (50.5\%). For this substudy, we further excluded 25 patients with a previous history of epilepsy (17 treated with diazepam), 1 patient who had a seizure before trial medication (in this case placebo) could be administered, and 33 patients (20 treated with diazepam) who had a transient ischemic attack. So, in total, 784 stroke patients remained for this substudy, 389 in the diazepam group (49.6\%) and 395 in the placebo group (50.4\%). Patient characteristics at baseline were similar in both groups (Table 1).

\section{Occurrence of Poststroke Seizures}

After 3 months of follow-up, 19 patients (2.4\% of the total patient group) had experienced at least one seizure. Of these, $10(53 \%)$ had their first seizure within the first 3 days (interval of trial medication). Fourteen patients (74\%) had their first seizure within 7 days after stroke (early seizures), and 5 patients (26\%) had their first seizure during the remaining follow-up period of 3 months. There was no statistically significant difference in seizure occurrence between patients treated with diazepam compared to the placebo group (1.5 vs. 3.3\%, IRR $0.45,95 \%$
CI: $0.18-1.16, p=0.11)$. However, when considering patients with a cortical infarct in the anterior circulation separately (so both TACI and PACI groups taken together), we found a significant difference in favor of diazepam treatment (Table 2). A seizure occurred in $0.9 \%$ of patients in the diazepam group versus $4.6 \%$ in the placebo group (IRR 0.20, 95\% CI: 0.05-0.78, $p=0.02$, NNT $=27$ ). In patients surviving beyond day 3 (the study treatment window), we also found that the rate of seizures was significantly lower in the diazepam group (0 patients) than in the placebo group (4 patients, $2.2 \%, \log$-rank test $p=$ $0.04)$.

\section{Occurrence of Pneumonia in the First 2 Weeks following Stroke}

Pneumonia developed in 113 patients (14\%). There was no difference in developing pneumonia between patients treated with diazepam compared to the placebo group (53 patients in the diazepam group [14\%] vs. 60 patients in the placebo group [15\%], $p=0.53$ ).

\section{Mortality}

During follow-up, 86 patients (11\%) died within 2 weeks. There was no difference between the diazepam 
Table 2. Number of patients with at least one poststroke seizure in the diazepam- and placebo-treated groups

\begin{tabular}{lrrrrrr}
\hline & Total PSS & Diazepam & Placebo & IRR & 95\% CI & $p$ value \\
\hline All 784 patients, 389 on diazepam & $19(2.4 \%)$ & $6(1.5 \%)$ & $13(3.3 \%)$ & 0.45 & $0.18-1.16$ & 0.11 \\
91 ICH patients, 43 on diazepam & $5(5.5 \%)$ & $2(4.7 \%)$ & $3(6.3 \%)$ & 0.75 & $0.13-4.44$ & 0.78 \\
693 IS patients, 346 on diazepam & $14(2.0 \%)$ & $4(1.2 \%)$ & $10(2.9 \%)$ & 0.39 & $0.13-1.18$ & 0.11 \\
408 PACI or TACI, 211 on diazepam & $11(2.7 \%)$ & $2(0.9 \%)$ & $9(4.6 \%)$ & 0.20 & $0.05-0.78$ & $\mathbf{0 . 0 2}$ \\
\hline
\end{tabular}

ICH, intracerebral hemorrhage; IS, ischemic stroke; PACI, partial anterior circulation infarction; TACI, total anterior circulation infarction; PSS, poststroke seizures; IRR, incidence rate ratio; 95\% CI, 95\% confidence interval. ${ }^{*}<0.05$ and statistically significant.

and the placebo group (39 patients died in the diazepam group [10\%] vs. 47 in the placebo group [12\%], OR 0.83; 95\% CI: $0.53-1.29, p=0.40)$. After 3 months, 153 patients had died (20\%), and again there was no difference between the diazepam and the placebo group (74 patients had died in the diazepam group [19\%] vs. 79 in the placebo group [20\%], OR 0.94; 95\% CI: 0.66-1.34, $p=$ $0.73)$.

Of the 113 patients who had developed pneumonia during the first 2 weeks following their stroke, 61 died during follow-up (54\%). This is significantly more than the 92 out of the 671 patients without pneumonia who died (14\%) (OR 7.38, 95\% CI: 4.80-11.35, $p<0.01$ ). When patients treated with diazepam and patients in the placebo group were analyzed separately, this difference appeared to be equally present in both groups. In patients treated with diazepam, 24 of 53 with pneumonia died $(45 \%)$ versus 50 of 336 without pneumonia (15\%) (OR 4.73 , 95\% CI: $2.55-8.79, p<0.01)$. In the placebo group, 37 of 60 with pneumonia died (62\%) versus 42 of 335 without pneumonia (13\%) (OR 11.22, 95\% CI: 6.08$20.71, p<0.01)$.

Of all patients who died, 5 had at least one seizure (3.3\%). Mortality in patients with seizures compared to patients without seizures showed no statistically significant difference (26\% died in the seizure group vs. $19 \%$ in the no-seizure group, OR 1.49, 95\% CI: 0.53-4.20, $p=$ $0.39)$.

\section{Previous Use of Benzodiazepines}

Of all patients, $90(11.5 \%)$ used a benzodiazepine before inclusion. In all cases, according to the EGASIS trial protocol, the benzodiazepines were withdrawn before randomization. Two of these patients, both randomized to the placebo group, experienced a seizure during follow-up, one after 12 days and one after 50 days.

Prevention of Poststroke Seizures

\section{Discussion}

We found a prophylactic effect of a 3-day treatment with diazepam directly after the stroke on the prevention of seizures in the first 3 months, but only in patients with an acute anterior circulation infarction. This effect has not been demonstrated previously in the sparse randomized studies on the effect of antiepileptic drugs on the occurrence of poststroke seizures [12]. The possible mechanism behind this effect of diazepam is the GABAergic stimulation. In the cascade of events which lead to the development of seizures, the GABAergic pathway is one of the changes which take place after cerebral stroke. Several animal studies show a potential antiepileptogenic effect [11].

In contrast to previous population-based studies [23$26]$, the frequency of seizures was rather low (3.2 and $6.3 \%$ as compared to $2.4 \%$ in our patients). This is striking because the registration of seizures was prospective, with active inquiries and a low loss to follow-up rate. It might be due to population effects in our study, with possibly less severely affected patients, which relates to a lower seizure risk. Unfortunately, because of the design of the primary study, which was not designed to study long-term seizure prevention, we did not have extensive information about seizures and seizure semiology. Furthermore, all seizures were clinician reported and we may have missed focal seizures which were missed during clinical follow-up. Thus, despite that seizures were a predefined outcome measure in the EGASIS study, these factors might have led to an underreporting of seizures as well as an underestimation of the effect of diazepam.

There are some limitations to this substudy. First, since we only have a 3-month follow-up, we do not cover the whole time frame of late seizures, which can occur as long as 2-3 years after the stroke. Second, another factor which might have affected our results is the obligatory 
withdrawal of previously used benzodiazepines, leading to a possible overestimation of seizure frequency. However, as none of the patients had a withdrawal seizure within 10 days after cessation of the benzodiazepine, we believe this effect is negligible.

Despite these limitations, the strengths of our study are the randomized, double-blind, placebo-controlled trial design, the start of the drug in the acute phase of stroke, and the large patient population. We also showed that treatment with diazepam in the acute phase of stroke is safe. The occurrence of pneumonia and mortality was similar in the diazepam and the placebo group. Although studying the effectiveness of diazepam in reducing the occurrence of poststroke seizures was not the primary aim of the EGASIS trial, our results suggest that a short treatment with diazepam may be useful as primary prevention of seizures in a subgroup of patients with stroke.

\section{Acknowledgements}

The authors thank the EGASIS study group for their contribution.

\section{Statement of Ethics}

This study received review board approval at each center, and written informed consent was obtained from all patients or their legal representative. The protocol was approved by the Medical Ethical Committee of Maastricht University/academic hospital Maastricht with No. MEC 99-063. The research was conducted in accordance with the Declaration of Helsinki.

\section{Conflict of Interest Statement}

None of the authors report conflicts of interest.

\section{Funding Sources}

The authors did not obtain additional funding sources for the present study.

\section{Author Contributions}

J.H. van Tuijl designed the study, gathered data, and wrote the draft of the manuscript. E.P.M. van Raak gathered data, performed the statistical analyses, and revised the manuscript for important intellectual content. R.J. van Oostenbrugge supervised the study and revised the manuscript for important intellectual content. A.P. Aldenkamp designed the study and revised the manuscript for important intellectual content. R.P.W. Rouhl designed and supervised the study and revised the manuscript for important intellectual content.

\section{Contribution to the Field Statement}

This study is one of the sparse studies on the prevention of seizures after stroke. As such, this substudy of a previously published multicenter, double-blind, randomized clinical trial aimed at demonstrating neuroprotective properties of the treatment under investigation provides valuable (though not perfect) data on the effect of a short treatment with diazepam in the acute phase of the stroke. It provides us with new arguments to consider treatment trials in a specific stroke patient group with a high risk of developing poststroke seizures. Prevention of poststroke seizures will probably increase quality of life in these patients.

\section{References}

1 Wang JZ, Vyas MV, Saposnik G, Burneo JG. Incidence and management of seizures after ischemic stroke: systematic review and metaanalysis. Neurology. 2017;89(12):1220-8.

2 Bogousslavsky J, Martin R, Regli F, Despland PA, Bolyn S. Persistent worsening of stroke sequelae after delayed seizures. Arch Neurol. 1992;49(4):385-8.

3 Berges S, Moulin T, Berger E, Tatu L, Sablot D, Challier B, et al. Seizures and epilepsy following strokes: recurrence factors. Eur Neurol. 2000;43(1):3-8.

4 De Reuck J, De Groote L, Van Maele G. Delayed transient worsening of neurological deficits after ischaemic stroke. Cerebrovasc Dis. 2006;22(1):27-32.

5 Vespa PM, O'Phelan K, Shah M, Mirabelli J, Starkman S, Kidwell C, et al. Acute seizures after intracerebral hemorrhage: a factor in progressive midline shift and outcome. Neurology. 2003;60(9):1441-6.
6 Kumral E, Uncu G, Dönmez I, Cerrahoglu Şirin T, Alpaydın S, Callı C, et al. Impact of poststroke seizures on neurological deficits: magnetic resonance diffusion-weighted imaging study. Eur Neurol. 2013;69(4):200-6.

7 Colon-Emeric CS, Pieper CF, Artz MB. Can historical and functional risk factors be used to predict fractures in community-dwelling older adults? Development and validation of a clinical tool. Osteoporos Int. 2002;13:95561.

8 Cloyd J, Hauser W, Towne A, Ramsay R, Mattson R, Gilliam F, et al. Epidemiological and medical aspects of epilepsy in the elderly. Epilepsy Res. 2006;68(Suppl 1):S39-48.

9 Winter Y, Daneshkhah N, Galland N, Kotulla I, Krüger A, Groppa S. Health-related quality of life in patients with poststroke epilepsy. Epilepsy Behav. 2018;80:303-6.

10 Loscher W, Brandt C. Prevention or modification of epileptogenesis after brain insults: experimental approaches and translational research. Pharmacol Rev. 2010;62:668-700.

11 Miziak B, Konarzewska A, Ułamek-Kozioł M, Dudra-Jastrzębska M, Pluta R, Czuczwar SJ. Anti-epileptogenic effects of antiepileptic drugs. Int J Mol Sci. 2020;21(7):2340.

12 Gilad R, Boaz M, Dabby R, Sadeh M, Lampl Y. Are post intracerebral hemorrhage seizures prevented by anti-epileptic treatment? Epilepsy Res. 2011;95(3):227-31.

13 Messe SR, Sansing LH, Cucchiara BL, Herman ST, Lyden PD, Kasner SE; CHANT investigators. Prophylactic antiepileptic drug use is associated with poor outcome following ICH. Neurocrit Care. 2009;11:38-44.

14 Consoli D, Bosco D, Postorino P, Galati F, Plastino M, Perticoni GF, et al. Levetiracetam versus carbamazepine in patients with late poststroke seizures: a multicenter prospective randomized open-label study (EpIC project). Cerebrovasc Dis. 2012;34(4):282-9. 
15 Sykes L, Wood E, Kwan J. Antiepileptic drugs for the primary and secondary prevention of seizures after stroke. Cochrane Database Syst Rev. 2014;1(1):CD005398.

16 Sheth KN, Martini SR, Moomaw CJ, Koch S, Elkind MS, Sung G, et al. Prophylactic antiepileptic drug use and outcome in the ethnic/ racial variations of intracerebral hemorrhage study. Stroke. 2015;46(12):3532-5.

17 van Tuijl JH, van Raak EP, de Krom MC, Lodder J, Aldenkamp AP. Early treatment after stroke for the prevention of late epileptic seizures: a report on the problems performing a randomised placebo-controlled doubleblind trial aimed at anti-epileptogenesis. Seizure. 2011;20(4):285-91.

18 Herman ST. Early poststroke seizures: is it time for prospective treatment trials? Neurology. 2011;77(20):1776-8.
19 Lodder J, van Raak L, Hilton A, Hardy E, Kessels A; EGASIS Study Group. Diazepam to improve acute stroke outcome: results of the early GABA-ergic activation study in stroke trial. a randomized double-blind placebocontrolled trial. Cerebrovasc Dis. 2006;21(12):120-7.

20 Pitkanen A, Kharatishvili I, Narkilahti S, Lukasiuk K, Nissinen J. Administration of diazepam during status epilepticus reduces development and severity of epilepsy in rat. Epilepsy Res. 2005;63:27-42.

21 Cunha AO, Mortari MR, Liberato JL, dos Santos WF. Neuroprotective effects of diazepam, carbamazepine, phenytoin and ketamine after pilocarpine-induced status epilepticus. Basic Clin Pharmacol Toxicol. 2009;104(6):470-7.

22 Bamford J, Sandercock P, Dennis M, Burn J, Warlow C. Classification and natural history of clinically identifiable subtypes of cerebral infarction. Lancet. 1991;337(8756):1521-6.

23 Procaccianti G, Zaniboni A, Rondelli F, Crisci $M$, Sacquegna T. Seizures in acute stroke: incidence, risk factors and prognosis. Neuroepidemiology. 2012;39(1):45-50

24 Alberti A, Paciaroni M, Caso V, Venti M, Palmerini F, Agnelli G. Early seizures in patients with acute stroke: frequency, predictive factors, and effect on clinical outcome. Vasc Health Risk Manag. 2008;4(3):715-20.

25 Beghi E, D’Alessandro R, Beretta S, Consoli D, Crespi V, Delaj L, et al. Incidence and predictors of acute symptomatic seizures after stroke. Neurology. 2011;77(20):1785-93.

26 Lancman ME, Golimstok A, Norscini J, Granillo R. Risk factors for developing seizures after a stroke. Epilepsia. 1993;34(1): $141-3$. 